\title{
THE SECOND-ORDER KLEIN-GORDON FIELD EQUATION
}

\section{GOMES and E. CAPELAS DE OLIVEIRA}

Received 22 June 2004

To Prof. Cesare M. G. Lattes on his eightieth birthday

\begin{abstract}
We introduce and discuss the generalized Klein-Gordon second-order partial differential equation in the Robertson-Walker space-time, using the Casimir second-order invariant operator written in hyperspherical coordinates. The de Sitter and anti-de Sitter space-times are recovered by means of a convenient choice of the parameter associated to the space-time curvature. As an application, we discuss a few properties of the solutions. We also discuss the case where we have positive frequency exponentials and the creation and annihilation operators of particles with known quantum numbers. Finally, we recover the Minkowskian case, that is, the case of null curvature.
\end{abstract}

2000 Mathematics Subject Classification: 81Q05, 35L05, 58J45, 33C05.

1. Introduction. The study of quantum field theories on a gravitational background can be seen as a theory for interacting quantized gravity and matter [1, 6]. In the context of linear theories, de Sitter and anti-de Sitter space-times are the most studied ones because, together with Minkowski space-time, they are the space-times which have maximal symmetry [16]. Here, we call our space-time the Robertson-Walker space-time, because the particular cases contained in our space-time are the de Sitter, anti-de Sitter, and Minkowski space-times, depending on the value of a certain parameter [8].

In a recent paper, Bros et al. [2] presented a study of quantum scalar fields on the de Sitter space-time based on analiticity in the complexified Riemannian manifold. More recently, Takook [15] discussed a covariant quantization of free spinor fields in a 4dimensional de Sitter space-time.

On the other hand, Notte Cuello and Capelas de Oliveira [10] discussed the Dirac wave equation in the de Sitter universe, using the factorization of the second-order Casimir invariant operator associated to the so-called Fantappié-de Sitter group. The same authors [11] presented and solved the Klein-Gordon and Dirac equations using spherical harmonics with spin weight.

This paper is organized as follows: in Section 1, we present the generalized KleinGordon wave equation in the Robertson-Walker space-time. In Section 2, we solve the second-order partial field equation using a convenient coordinate system which separates the angular part, where the solution is given by hyperspherical harmonics, and another partial differential equation containing the separated radial and temporal parts. It is worth noting that other coordinate systems are possible. For example, Capelas de Oliveira et al. [3] used canonical hyperspherical coordinates to solve the generalized Klein-Gordon second-order partial differential wave equation in the $n$-dimensional de 
Sitter space-time and Redmount and Takagi [13] resorted to the so-called Rindler hyperspherical coordinates to discuss a field theory in de Sitter space-time for a free and massless field embedded in a flat Minkowskian space-time. Polarski [12] discussed the scalar wave equation on static de Sitter and anti-de Sitter spaces with four dimensions, using polyspherical coordinates.

In Section 3, we present some properties of the function, a hypergeometric function, which is a solution of the ordinary differential equation associated to the mass and dimensionality terms and reobtain the classical result where Chernikov and Tagirov [5] construct the quantum theory for a scalar field in de Sitter universe. We also recover, as a particular case, the positive frequency exponentials in a 4-dimensional space-time which were obtained by Tagirov [14] and by Wyrozumski [17], where the latter discusses an alternative construction of the vacuum in a (1+3)-dimensional de Sitter space-time. Finally, we present our conclusions and perspectives.

2. Generalized Klein-Gordon wave equation. In this section, we present and discuss the generalized Klein-Gordon wave equation in the Robertson-Walker space-time.

We call a generalized Klein-Gordon partial second-order differential equation a KleinGordon wave equation, which is obtained using the Casimir second-order invariant operator associated to the so-called Fantappié-de Sitter group [8], that is,

$$
A^{2}\left\{\partial_{i}^{2}+\frac{\bar{k}}{r_{0}^{2}} x_{i} x_{j} \partial_{i} \partial_{j}+2 \frac{\bar{k}}{r_{0}^{2}} x_{i} \partial_{i}\right\} \Psi\left(x_{i}\right)=\frac{\bar{k}}{r_{0}^{2}} \Omega^{2} \Psi\left(x_{i}\right),
$$

with $i, j=0,1,2, \ldots, d+2$. Here, $r_{0}$ is the radius of the universe and $A^{2}$ is the so-called Cayley-Klein absolute given by

$$
A^{2}=1+\frac{\bar{k}}{r_{0}^{2}}\left(x_{i}^{2}+x_{0}^{2}\right)
$$

with $i=1,2, \ldots, d+2$. In order to have a physical meaning, we take $x_{0}=i c t$ where $c$ is the speed of light and $\tau$ is the time. The constant in the second member is given by

$$
\Omega^{2}=\bar{k}\left(\frac{m c}{\hbar} r_{0}\right)^{2}+\frac{(d+1)(d+3)}{4} .
$$

The constants $m$ and $\hbar$ have the usual meaning and the second term on the right-hand side, where $d$ denotes the dimension, is due to the curvature. The parameter $\bar{k}=0,1,-1$ is associated to the Minkowski, de Sitter, and anti-de Sitter space-times, respectively. If we consider $\bar{k}=-1$, we have the $\mathrm{SO}(3,2)$ group; $\bar{k}=1$, the $\mathrm{SO}(4,1)$ group, and $\bar{k}=0$, the Poincaré group associated to their respective space-times.

To simplify our partial differential equation, we introduce the variables

$$
\sqrt{\bar{k}} \frac{x_{i}}{r_{0}}=\rho, \quad \sqrt{\bar{k}} \frac{c \tau}{r_{0}}=t,
$$


obtaining the partial differential equation

$$
\left\{\partial_{i}^{2}+\rho_{i} \rho_{j} \partial_{i} \partial_{j}+2 \rho_{i} \partial_{i}\right\} \Psi\left(\rho_{i}\right)=\frac{\Omega^{2}}{A^{2}} \Psi\left(\rho_{i}\right),
$$

where $i, j=0,1,2, \ldots, d+2, A^{2}=1+\rho_{i}^{2}-t^{2}$ and $\Omega^{2}$ as given above.

To solve this partial differential equation, we can introduce the canonical hyperspherical coordinates [3] but the separated equations contain a term of mixed derivatives. Thus we use here the following system of coordinates:

$$
\begin{aligned}
i \rho_{0} & =\tan \tau \sec \xi, \\
\rho_{1} & =\tan \xi \cos \theta_{1}, \\
\rho_{2} & =\tan \xi \sin \theta_{1} \cos \theta_{2}, \\
\rho_{3} & =\tan \xi \sin \theta_{1} \sin \theta_{2} \cos \theta_{3}, \\
\vdots & \quad \vdots \\
\rho_{d} & =\tan \xi \sin \theta_{1} \cdots \sin \theta_{d-1} \cos \theta_{d}, \\
\rho_{d+1} & =\tan \xi \sin \theta_{1} \cdots \sin \theta_{d-1} \sin \theta_{d} \cos \phi, \\
\rho_{d+2} & =\tan \xi \sin \theta_{1} \cdots \sin \theta_{d-1} \sin \theta_{d} \sin \phi,
\end{aligned}
$$

$0 \leq \theta_{j} \leq \pi$ with $j=1,2, \ldots, d$ and $0 \leq \phi \leq 2 \pi$. We then get, after a separation of variables, the regular solution of the equation obtained, for the angular part, as follows:

$$
e^{ \pm i m_{d}} \phi \prod_{k=0}^{d-1}\left(\sin \theta_{k+1}\right)^{m_{k+1}} C_{m_{k}-m_{k+1}}^{m_{k+1}+(d-k) / 2}\left(\cos \theta_{k+1}\right),
$$

where $C_{\mu}^{\nu}(x)$ are the Gegenbauer polynomials of degree $\mu$ and order $v$ and $n=m_{0} \geq$ $m_{1} \geq \cdots \geq m_{d} \geq 0$.

As for the partial differential equation in the $\xi$ and $\tau$ variables, we can write

$$
\begin{aligned}
& {\left[\frac{\partial^{2}}{\partial \xi^{2}}+(d+1) \cot \xi \frac{\partial}{\partial \xi}+\cos ^{2} \tau \frac{\partial^{2}}{\partial \tau^{2}}\right.} \\
& \left.-(d+2) \sin \tau \cos \tau \frac{\partial}{\partial \tau}-\frac{n(n+d)}{\sin ^{2} \xi}-\Omega^{2} \cos ^{2} \tau\right] \Psi(\xi, \tau)=0,
\end{aligned}
$$

where $n=0,1,2, \ldots$ (some authors call (2.8), a partial differential equation, the $r a$ dial differential equation, because the other partial differential equation contains the angular part only).

3. Solution of the field equation. In Section 1, we presented the generalized KleinGordon field equation and separated the angular equation which admits the hyperspherical harmonics as solution (in [3], we use canonical hyperspherical coordinates and we obtain the same angular equation, but the remaining differential equation is different). Here, we solve the equation in the $\xi$ and $\tau$ variables. With this aim, we introduce another separation of variables

$$
\Psi(\xi, \tau)=R(\xi) T(\tau)
$$


and we obtain two ordinary differential equations as follows:

$$
\begin{gathered}
{\left[\frac{d^{2}}{d \xi^{2}}+(d+1) \cot \xi \frac{d}{d \xi}-\frac{n(n+d)}{\sin ^{2} \xi}-\Lambda\right] R(\xi)=0} \\
{\left[\cos ^{2} \tau \frac{d^{2}}{d \tau^{2}}-(d+2) \sin \tau \cos \tau \frac{d}{d \tau}-\Omega^{2} \cos ^{2} \tau+\Lambda\right] T(\tau)=0,}
\end{gathered}
$$

where $\Lambda$ is a constant.

Introducing $\Lambda=s(s+d+1)$ with $s=0,1,2, \ldots$, we can write the solution of the equation in the $\xi$ variable in terms of the hypergeometric function, that is,

$$
R(\xi)=A \sin ^{n} \xi_{2} F_{1}\left(n-s, n+s+d+1 ; n+\frac{d+2}{2} ; \sin ^{2} \frac{\xi}{2}\right),
$$

where $A$ is a constant.

In the case that considers the de Sitter universe, this solution can be written in terms of the Gegenbauer polynomials

$$
T(\xi)=\sin ^{n} \xi C_{s-n}^{n+(d+1) / 2}(\cos \xi),
$$

where $s-n \geq 0$ and $C_{\mu}^{\nu}(x)$ are the Gegenbauer polynomials.

Finally, we must solve the following ordinary differential equation:

$$
\left[\frac{d^{2}}{d \tau^{2}}-(d+2) \tan \tau \frac{d}{d \tau}-\frac{s(s+d+1)}{\cos ^{2} \tau}-\Omega^{2}\right] T(\tau)=0,
$$

where $\Omega^{2}$ is given by (2.3).

To solve this ordinary differential equation, we introduce the change of variable

$$
T(\tau)=\cos ^{\mathcal{S}} \tau F(\tau)
$$

and we obtain

$$
\left\{t(1-t) \frac{d^{2}}{d t^{2}}+\left[s+\frac{d+3}{2}-(2 s+d+3) t\right] \frac{d}{d t}-s(s+d+2)-\Omega^{2}\right\} F(t)=0,
$$

where $2 t=1-\sin \tau$.

The solution of the equation in variable $\tau$ can be obtained in terms of the hypergeometric function and then

$$
T(\tau)=\cos ^{s} \tau_{2} F_{1}\left(v+s+\frac{d+1}{2},-v+s+\frac{d+3}{2} ; s+\frac{d+3}{2} ; \frac{1-\sin \tau}{2}\right),
$$

where $2 v=1-\sqrt{1-4 M^{2}}$ and $M^{2}=\bar{k}\left(m c r_{0} / \hbar\right)^{2}$. Using the theory of hypergeometric functions, we can obtain the second linearly independent solution.

4. Some properties of the solutions. To discuss a few properties of the solutions of (3.5), it is convenient to introduce a change of independent variable of the type

$$
\sin \tau=i \tan \beta \text {. }
$$


We then obtain the equation

$$
\left[\frac{d^{2}}{d \beta^{2}}+(d+1) \tan \beta \frac{d}{d \beta}+s(s+d+1)+\frac{\Omega^{2}}{\cos ^{2} \beta}\right] T(\beta)=0 .
$$

Finally, introducing another change of dependent variable

$$
T(\beta)=\cos ^{(d+1) / 2} \beta F(\beta),
$$

we can write

$$
\left[\frac{d^{2}}{d \beta^{2}}+\left(q^{2}+\frac{M^{2}}{\cos ^{2} \beta}\right)\right] F(\beta)=0,
$$

where $q=s+(d+1) / 2$. This ordinary differential equation is the same equation obtained by Chernikov and Tagirov [5] using another procedure. Two solutions of the ordinary differential equation are given by [4]

$$
F_{q v}^{ \pm}(\beta)=A(q, v) e^{ \pm i q \beta}{ }_{2} F_{1}\left(v, 1-v ; q+1 ; \frac{1 \pm i \tan \beta}{2}\right),
$$

where $A(q, v)$ is a normalization constant and ${ }_{2} F_{1}(a, b ; c ; x)$ is the hypergeometric function. We note that the equation in the $\beta$ variable is invariant under the change $\beta \rightarrow-\beta$ and the two solutions are linearly independent.

We list below some properties of the above hypergeometric function (solutions of the differential equation):

(a) complex conjugate: $\left[F_{q v}^{+}(\beta)\right]^{*}=F_{q v}^{-}(\beta)$,

(b) parity: $F_{q v}^{+}(\beta)=F_{q v}^{-}(-\beta)$,

(c) Wronskian: $W\left[F_{q v}^{+}(\beta), F_{q v}^{-}(\beta)\right]=2 / i$,

(d) free wave: $F_{q 0}^{ \pm}(\beta) \propto e^{ \pm i q \beta}$.

In [5], the authors give a complete list of the properties of this function and discuss the field commutator and the transition to second quantization.

To finish this section, we introduce the change of variable $\sin \tau=i \cot \beta$, in (3.5) and we get

$$
\left\{\frac{d^{2}}{d \beta^{2}}-(d+1) \cot \beta \frac{d}{d \beta}+s(s+d+1)+\frac{\Omega^{2}}{\sin ^{2} \beta}\right\} T(\beta)=0 .
$$

The solution of the above equation can be written as follows:

$$
T(\beta)=\sin ^{(d+2) / 2} \beta P_{s+d / 2}^{\ell}(\cos \beta),
$$

and where $\ell$ and $\Omega$ are related by the expression

$$
\left(\frac{d+2}{2}\right)^{2}-\Omega^{2}=\ell^{2}
$$

where $P_{\nu}^{\mu}(x)$ are associated Legendre functions. 
We now consider the case $d=1$. Taking $\ell=-N-3 / 2$, where $N$ is an integer, and using a relation involving the associated Legendre functions [9], we obtain

$$
T(\beta)=\sin ^{3 / 2} \beta\left\{P_{s+1 / 2}^{N+3 / 2}(\cos \beta)-\frac{2 i}{\pi} Q_{s+1 / 2}^{N+3 / 2}(\cos \beta)\right\},
$$

which are proportional to the positive-frequency solutions (to write the positive-frequency solutions, we take $d=1$ in (2.7) and (3.4) and use the equations for $T(\beta)$ ). These solutions are the same solutions obtained by Chernikov and Tagirov in [5], where they discuss the creation and annihilation operators of particles with known quantum numbers, and by Wyrozumski [17] using the methodology of Fourier transform. Finally, if we consider $\bar{k}=0$ or $r_{0} \rightarrow \infty$, we recover the Minkowskian case (Klein-Gordon equation in the Robertson-Walker universe, private communication, 2004).

5. Conclusions and perspectives. In this paper, we discussed the second-order field equation associated to the Robertson-Walker space-time, that is, the generalized KleinGordon wave equation, using an alternative methodology, by means of Casimir secondorder invariant operator. We solved this equation using a convenient system of coordinates. We separated the differential equation in its angular part, solved in terms of the Gegenbauer polynomials, and another partial differential equation which is solved in terms of hypergeometric functions. A few properties of this particular hypergeometric function were presented. As a particular case, when we have $d=1$ (4-dimensional case) we recovered Chernikov and Tagirov's as well as Wyrozumski's results, that is, the positive-frequency solutions, which were obtained with different procedures. Finally, we note that for $\ell= \pm 1 / 2$ we have $M=0$ and then the second-order field equation is conformal invariant.

A natural continuation of this paper is the calculation of the solution of Dirac equation by means of the factorization method, using the spin weight operators, associated to the Casimir second-order invariant operator (on the first-order field equation, private communication, 2001). On the other hand, we can discuss the polynomial solutions of the generalized Laplace differential equation, which depend on the dimension. When we considered $d=1$, we recovered our recent result [7].

ACKNOWLEDGMENTS. The second author is grateful to Professor Jorge Rezende for the invitation to visit Lisbon University, where this manuscript was finished. We are grateful to Dr. J. Emílio Maiorino for many and useful discussions.

\section{REFERENCES}

[1] N. D. Birrell and P. C. W. Davies, Quantum Fields in Curved Space, Cambridge Monographs on Mathematical Physics, vol. 7, Cambridge University Press, Cambridge, 1982.

[2] J. Bros, J.-P. Gazeau, and U. Moschella, Quantum field theory in the de Sitter universe, Phys. Rev. Lett. 73 (1994), no. 13, 1746-1749.

[3] E. Capelas de Oliveira, G. Arcidiacono, and E. A. Notte Cuello, The generalized Klein-Gordon wave equation, Hadronic J. Suppl. 13 (1998), no. 3, 249-256.

[4] E. Capelas de Oliveira and J. Emílio Maiorino, Introduction to the Methods of Applied Mathematics, 2nd ed., Editora da Unicamp, São Paulo, 2003. 
[5] N. A. Chernikov and E. A. Tagirov, Quantum theory of scalar field in de Sitter space-time, Ann. Inst. H. Poincaré Sect. A (N.S.) 9 (1968), 109-141.

[6] S. A. Fulling, Aspects of Quantum Field Theory in Curved Space-Time, London Mathematical Society Student Texts, vol. 17, Cambridge University Press, Cambridge, 1989.

[7] D. Gomes and E. Capelas de Oliveira, On a new class of polynomials, Hadronic J. Suppl. 13 (1998), no. 4, 383-393.

[8] _ Wave equations in the de Sitter universe, TEMA Tend. Mat. Apl. Comput. 4 (2003), no. 1, 51-60 (Portuguese).

[9] W. Magnus, F. Oberhettinger, and R. P. Soni, Formulas and Theorems for the Special Functions of Mathematical Physics, Springer-Verlag, New York, 1966.

[10] E. A. Notte Cuello and E. Capelas de Oliveira, Dirac wave equation in the de Sitter universe, Internat. J. Theoret. Phys. 36 (1997), no. 5, 1231-1247.

[11] Klein-Gordon and Dirac equations in de Sitter space-time, Internat. J. Theoret. Phys. 38 (1999), no. 2, 585-598.

[12] D. Polarski, The scalar wave equation on static de Sitter and anti-de Sitter spaces, Classical Quantum Gravity 6 (1989), no. 6, 893-900.

[13] I. H. Redmount and S. Takagi, Hyperspherical Rindler space, dimensional reduction, and de Sitter-space scalar field theory, Phys. Rev. D (3) 37 (1988), no. 6, 1443-1455.

[14] E. A. Tagirov, Consequences of field quantization in de Sitter type cosmological models, Ann. Physics 76 (1973), 561-579.

[15] M. V. Takook, Spin 1/2 field theory in the de Sitter space-time, preprint, 2000, http://arxiv. org/abs/gr-qc/0005077.

[16] S. Weinberg, Gravitation and Cosmology: Principles and Applications of the General Theory of Relativity, John Wiley \& Sons, New York, 1972.

[17] T. Wyrozumski, On an alternative construction of the vacuum in $(1+3)$-dimensional de Sitter spacetime, Classical Quantum Gravity 5 (1988), no. 12, 1607-1613.

D. Gomes: Departamento de Matemática, Universidade Federal de Santa Maria, 97119-900 Santa Maria, Rio Grande do Sul, Brazil

E-mail address: deni1 son@ccne.ufsm.br

E. Capelas de Oliveira: Grupo de Física-Matemática, Faculdade de Ciências, Universidade de Lisboa, 1649-003 Lisboa, Portugal

E-mail address: ecape1as@ci i .fc.u1.pt

Current address: Departamento de Matemática Aplicada, Universidade Estadual de Campinas, 13083-970 Campinas, São Paulo, Brazil

E-mail address: cape1as@ime.unicamp.br 


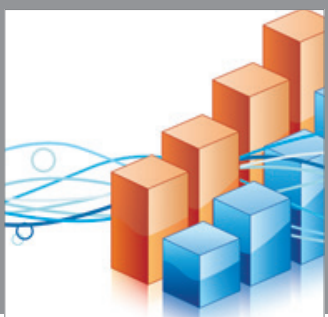

Advances in

Operations Research

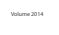

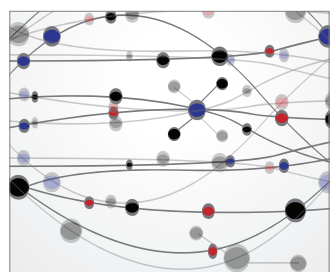

\section{The Scientific} World Journal
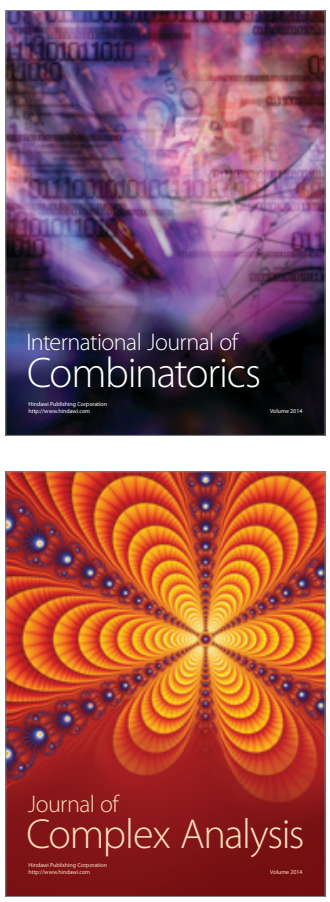

International Journal of

Mathematics and

Mathematical

Sciences
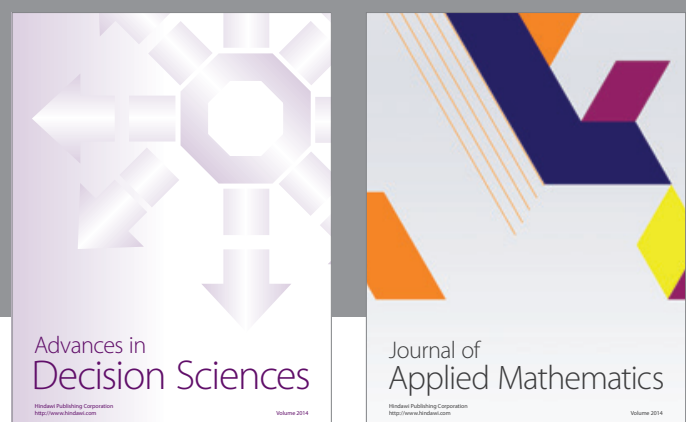

Journal of

Applied Mathematics
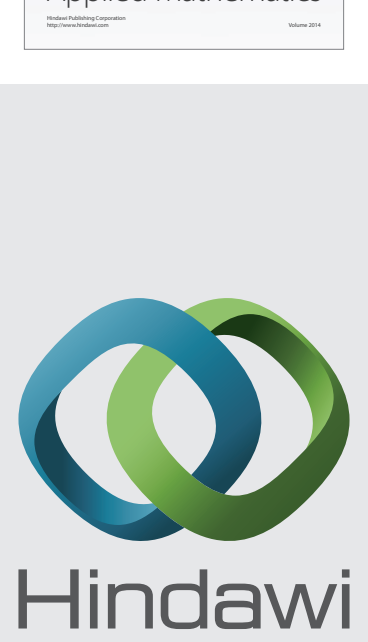

Submit your manuscripts at http://www.hindawi.com
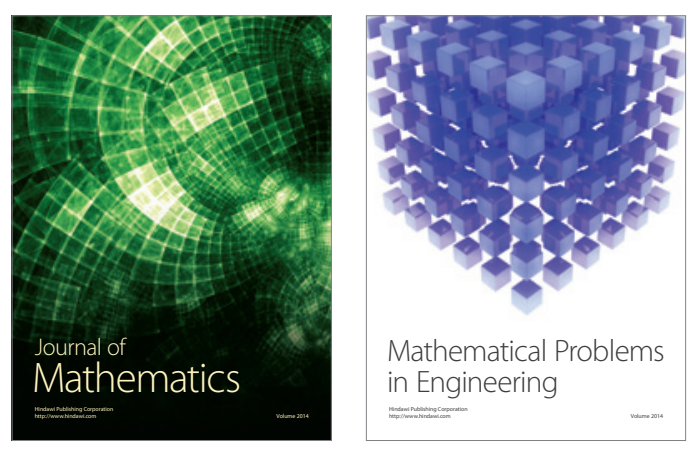

Mathematical Problems in Engineering
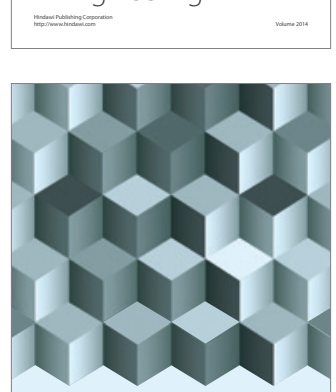

Journal of

Function Spaces
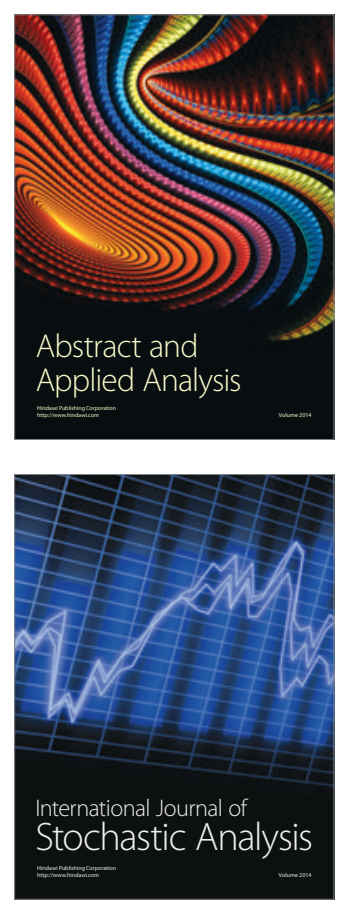

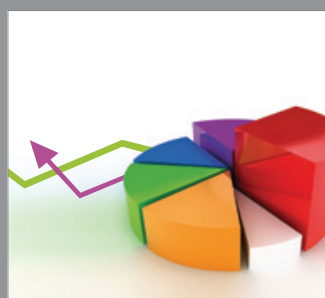

ournal of

Probability and Statistics

Promensencen
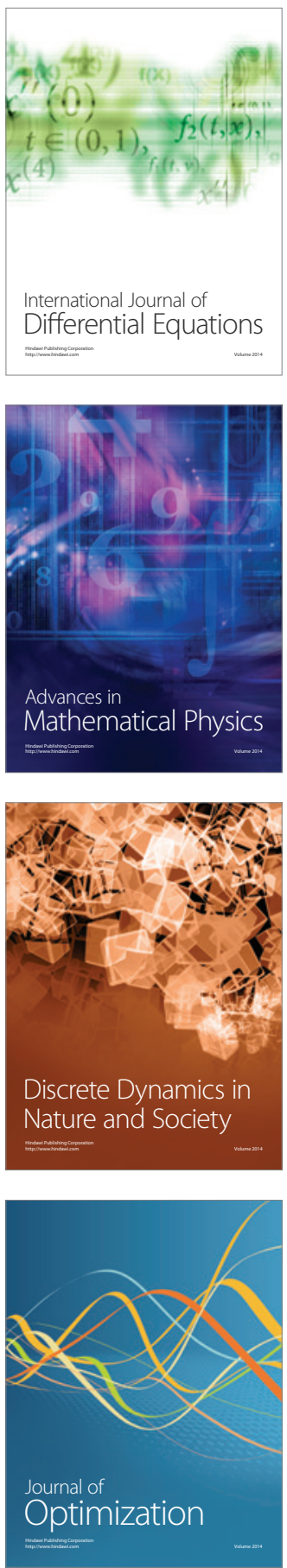